\title{
MODERN ASPECTS OF CONVERGENCE THEORY
}

\section{JEMAL KHARITONASHVILI}

PhD in Economics, Associate Professor

Ivane Javakhishvili Tbilisi State University, Georgia

jemal.kharitonashvili@tsu.ge

Abstract. In the 50 s and 60 s of the twentieth century, when a proper parity was established in the economic rivalry between the capitalist and socialist systems, the economists and sociologists in the bourgeois reformist current formulated the theory of convergence ("convergence" comes from Latin convergere - from con- 'together' + vergere - 'incline'). Convergence implies to merge, to bring two different socio-economic systems (capitalism and communism) together and to create a common techno-industrial system. Convergence involves similarity and integration of some characteristic features of different social and political systems and their structures, which gradually become similar and by means of interrelationship, cooperation and mutual understanding overcome the obstacles and acquire common character and become especially active in the conditions of scientific and technical development, internationalization and globalization until they create a new type, mixed, i.e. hybrid society, which includes the positive features of both capitalism and socialism. The global problems common to all mankind must be solved according to the above said.

\section{KEYWORDS: CONVERGENCE, SYNTHESIS, PLURALISM, MERITOCRACY.}

For citation: Kharitonashvili, J., (2021). Modern Aspects of Convergence Theory. Globalization and Business. 12, 57-60. (In Georgian). https://doi.org/10.35945/gb.2021.12.007 


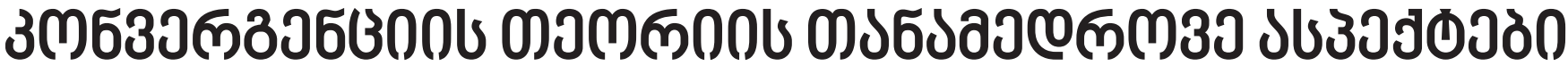

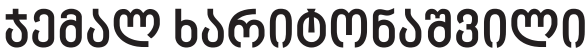

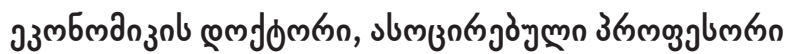

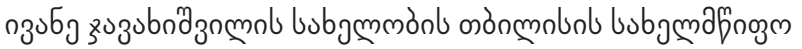

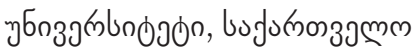

jemal.kharitonashvili@tsu.ge

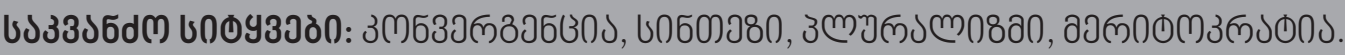

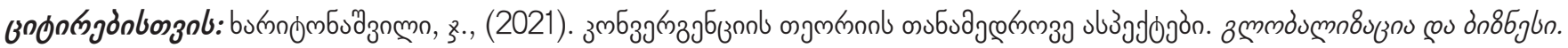
12, 57-60. https://doi.org/10.35945/gb.2021.12.007

\section{эอเวз১ตั0}

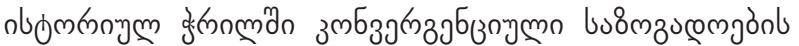

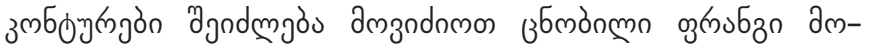

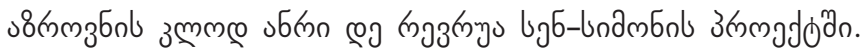

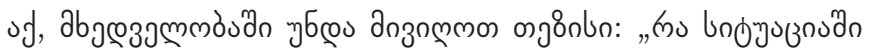

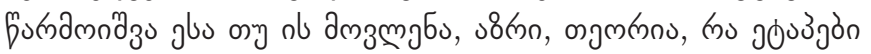

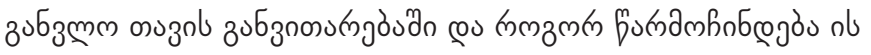

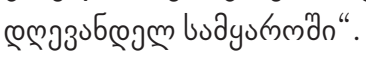

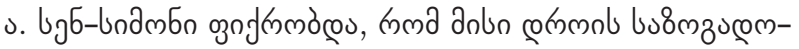

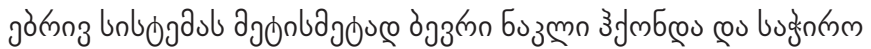

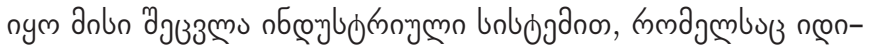

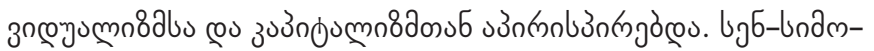

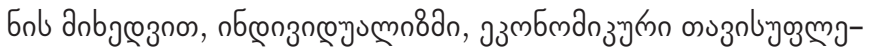

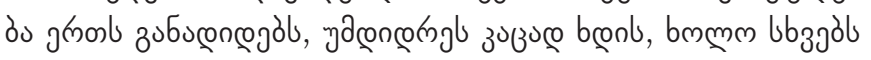

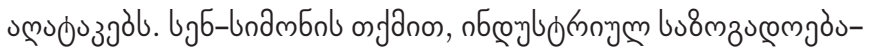

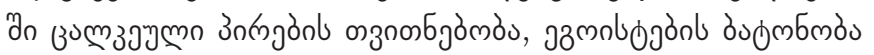

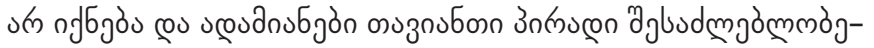

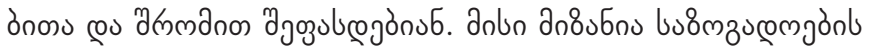

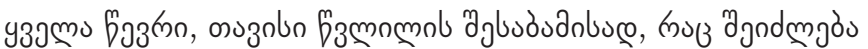

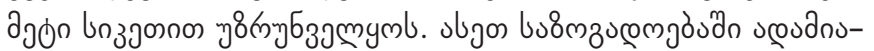

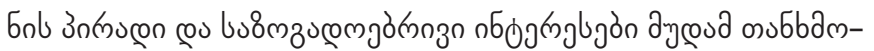

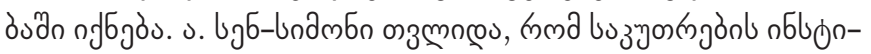

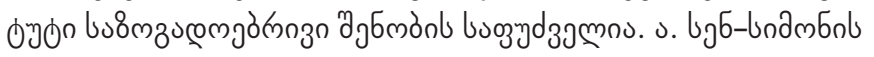

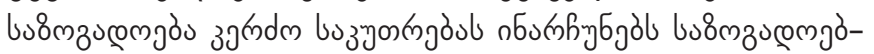

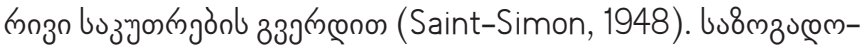

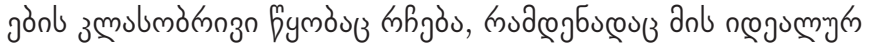

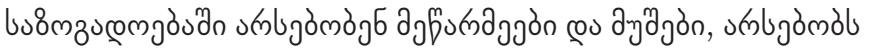

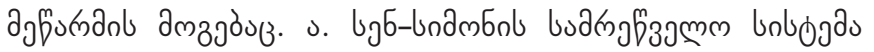

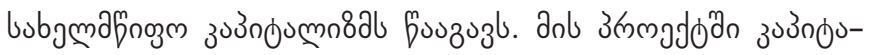

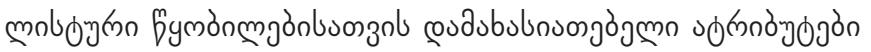

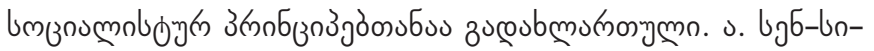

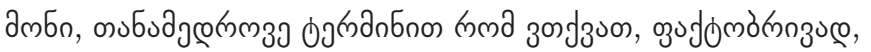

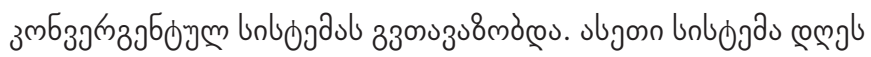

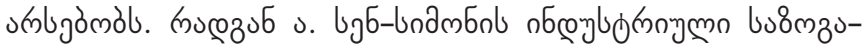

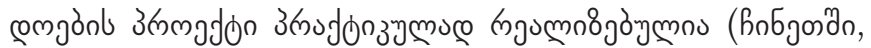

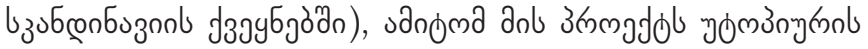

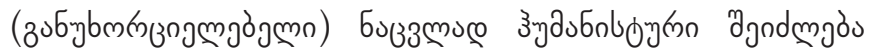

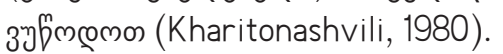

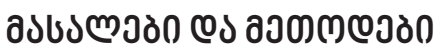

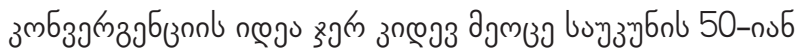

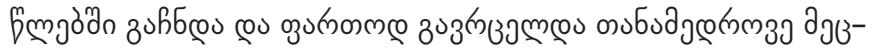

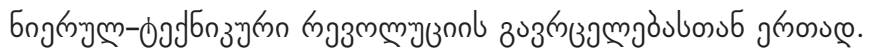

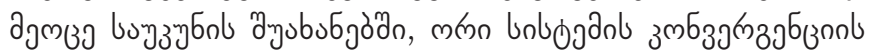

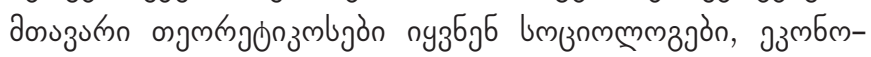

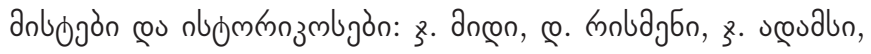

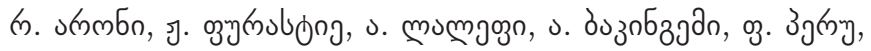

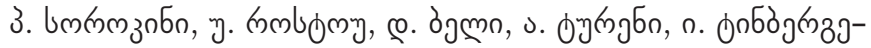

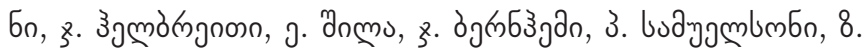

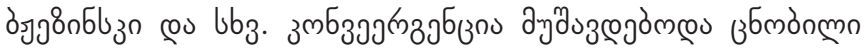

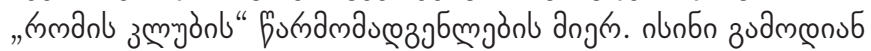

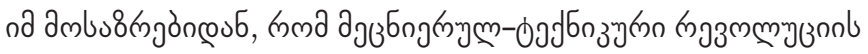

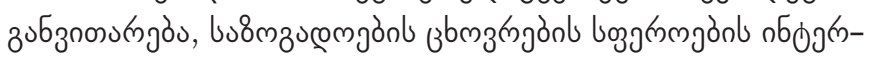

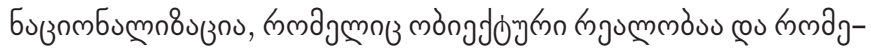

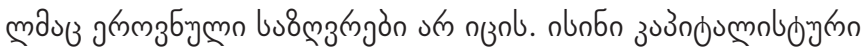

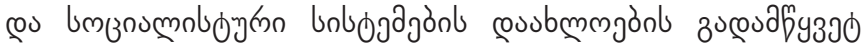

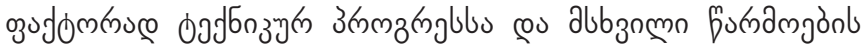

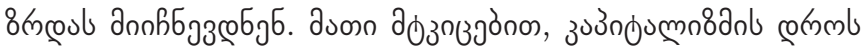

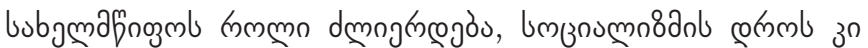

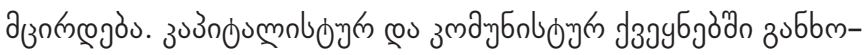

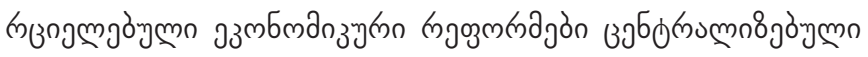

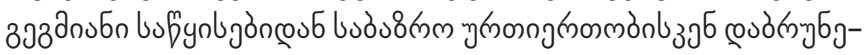
oul dmulbusjòcos. 
ง. азzпб mnym bnlojagal

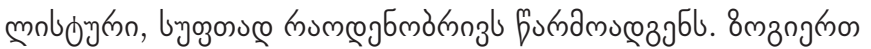

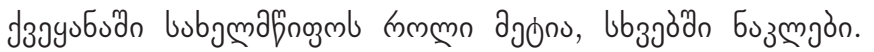

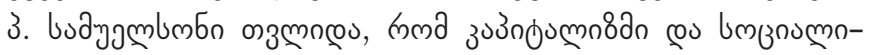

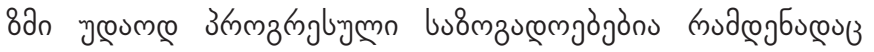

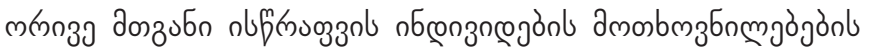

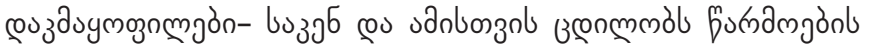

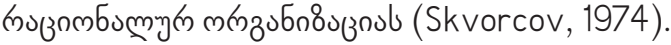

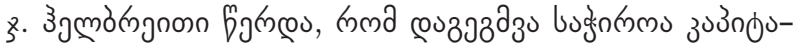
molby

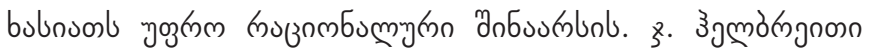

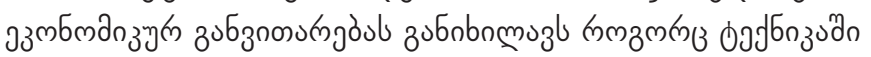

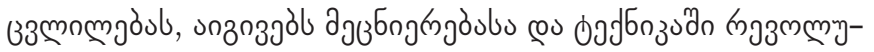

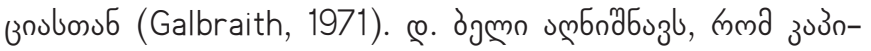

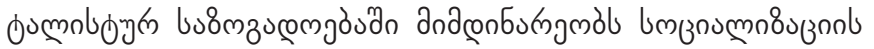

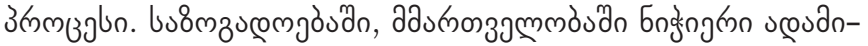

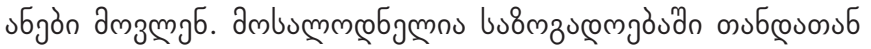

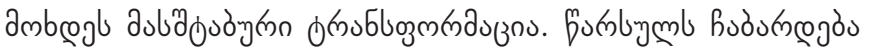

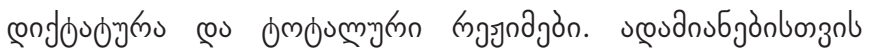

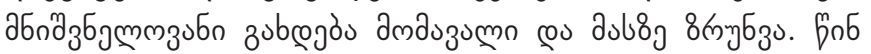

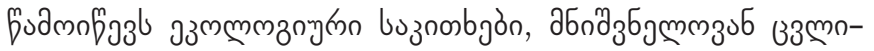

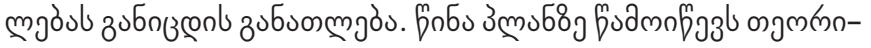

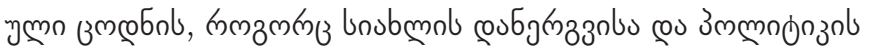

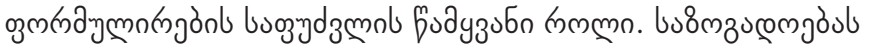

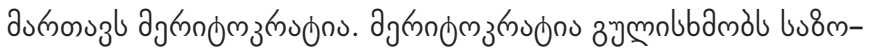

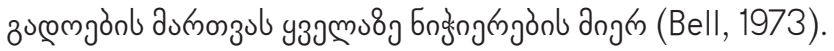

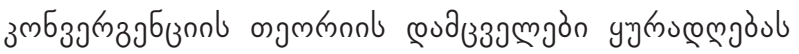

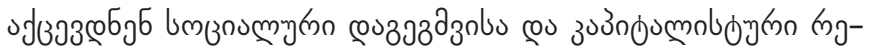

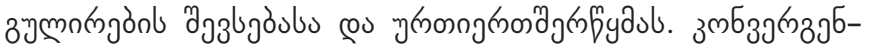

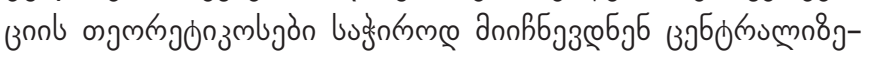

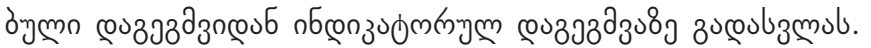

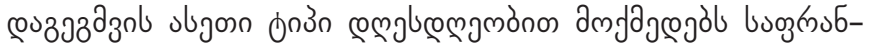

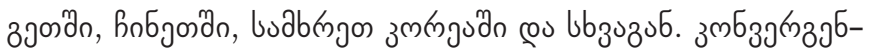

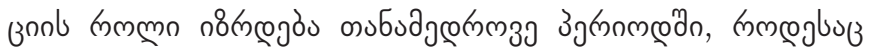

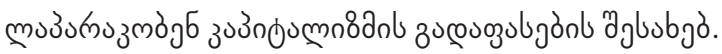

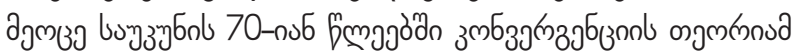

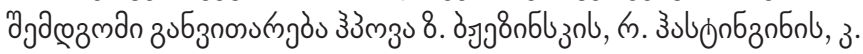

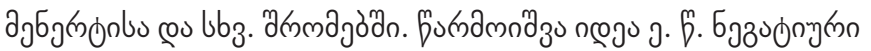
змб

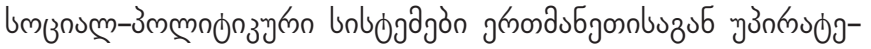

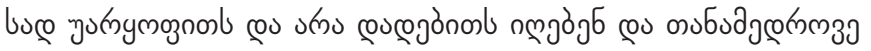

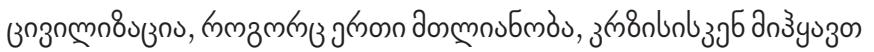

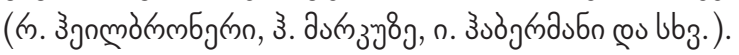

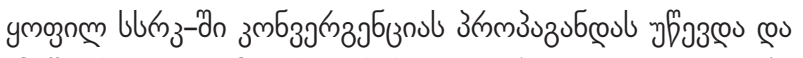

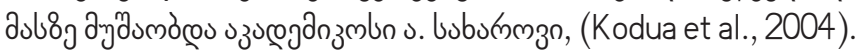

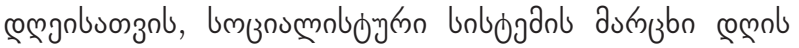

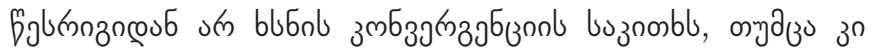

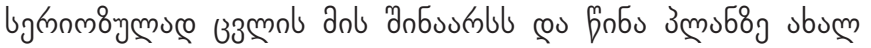

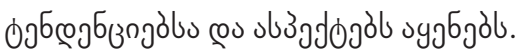

\section{esu335s}

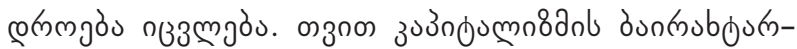

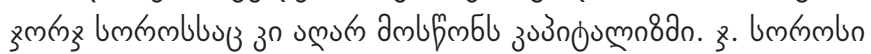
anyonnogab: „, „b

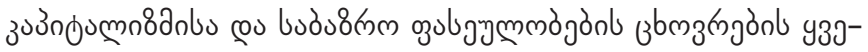

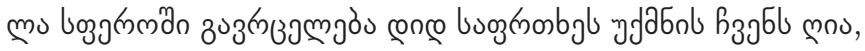

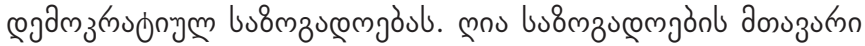
a

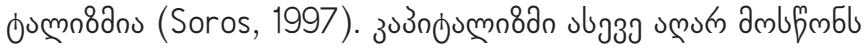

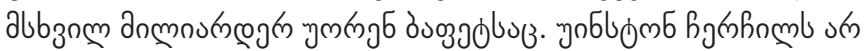

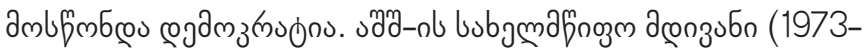

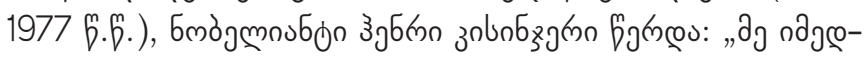

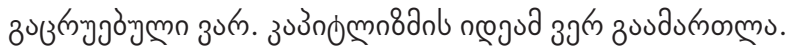

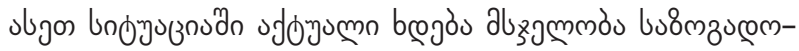

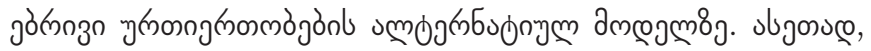

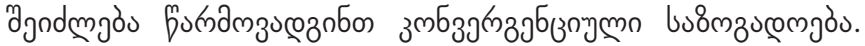

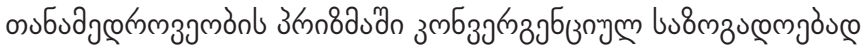

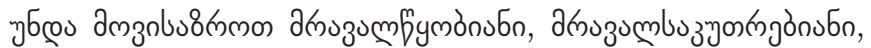

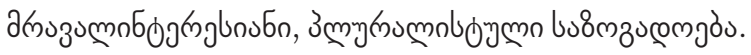

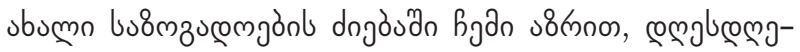

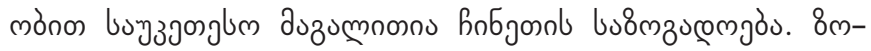

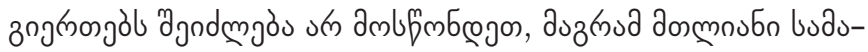

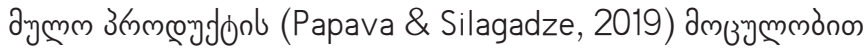

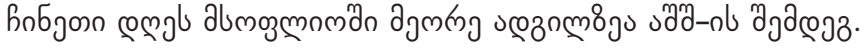

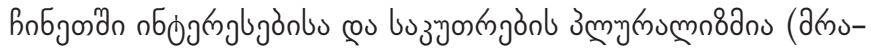

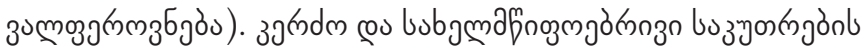

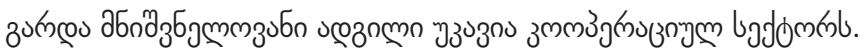

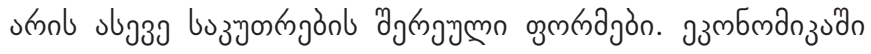

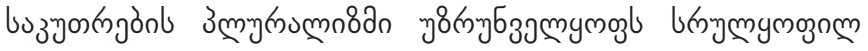

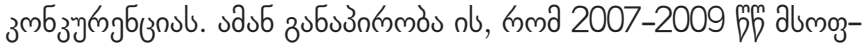

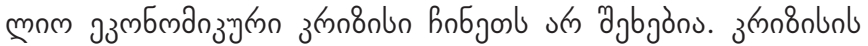

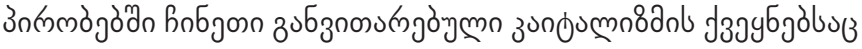

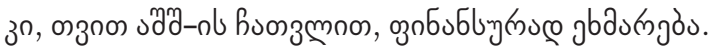

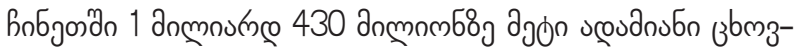

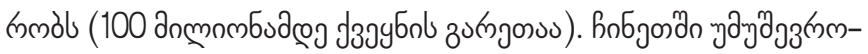

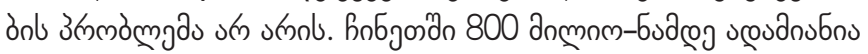

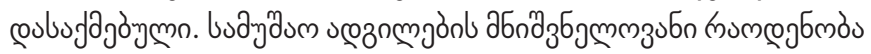

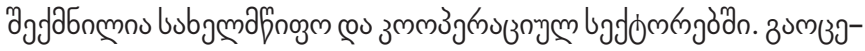

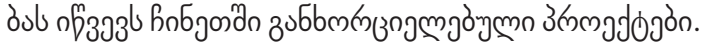

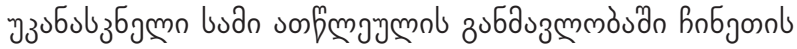

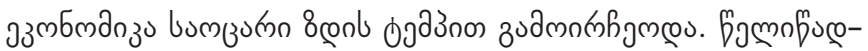

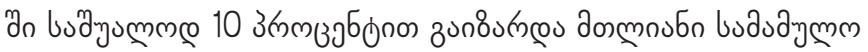

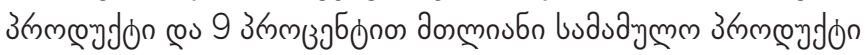

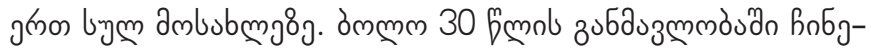

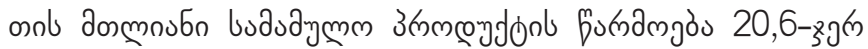

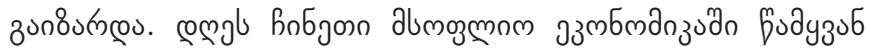

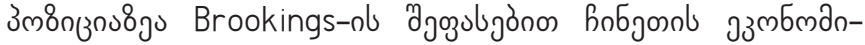

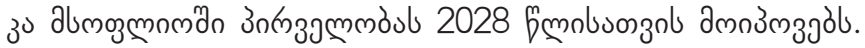

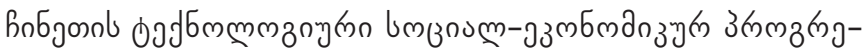




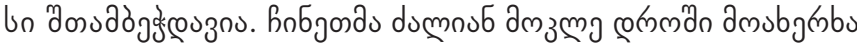

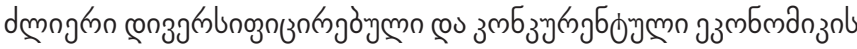

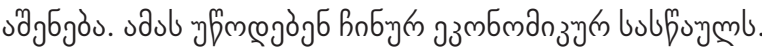

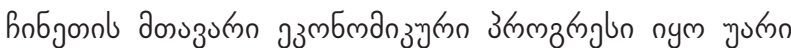

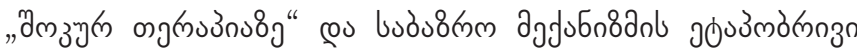

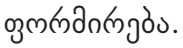

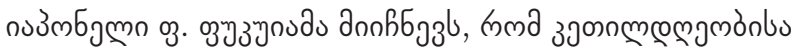

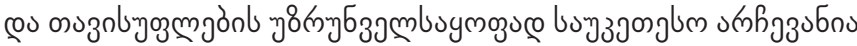

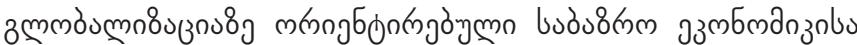

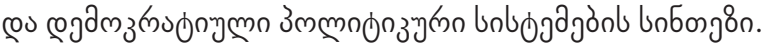

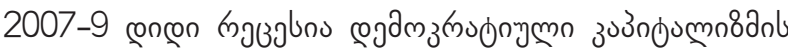

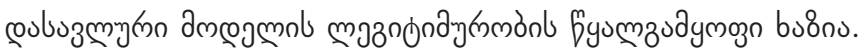

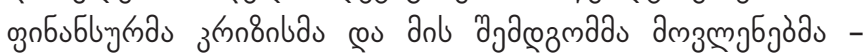

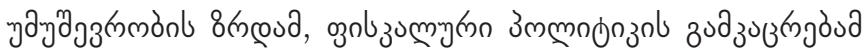

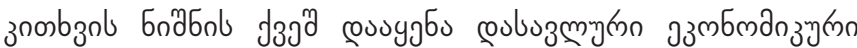

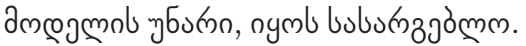

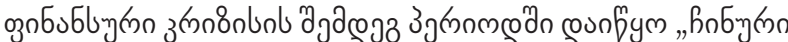

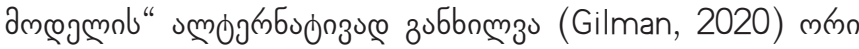

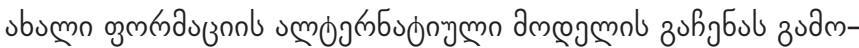
ymozl,

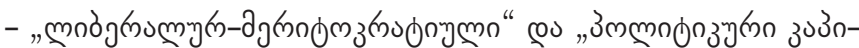

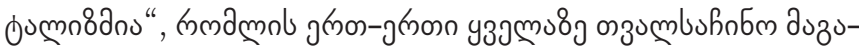

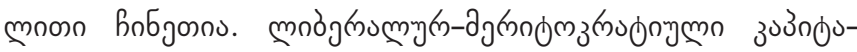

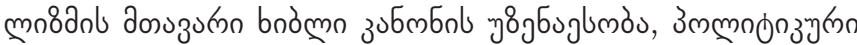

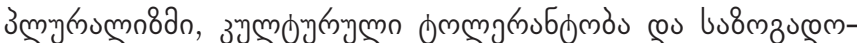

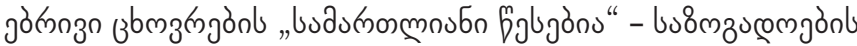

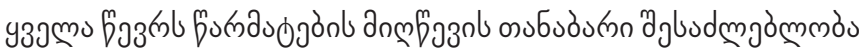

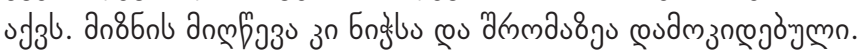

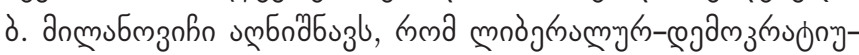

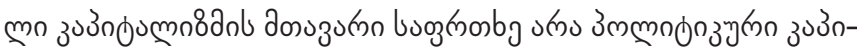

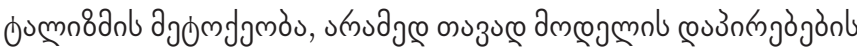

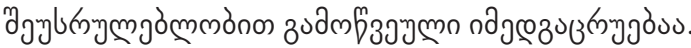

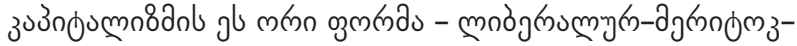

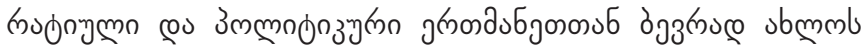

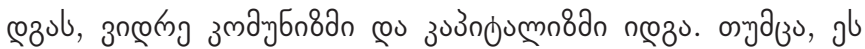

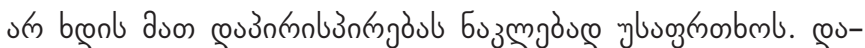

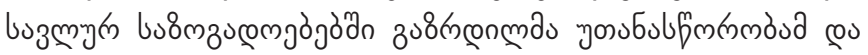

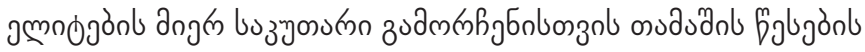

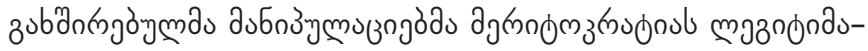

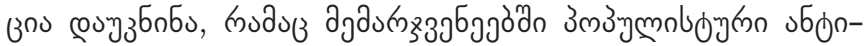

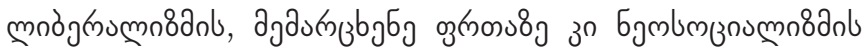

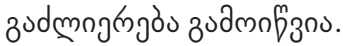

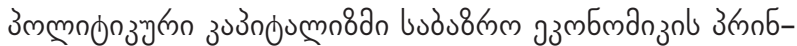

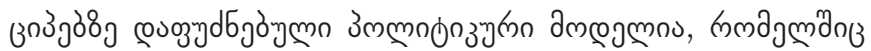

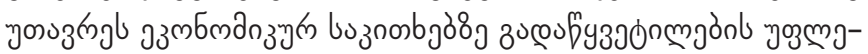

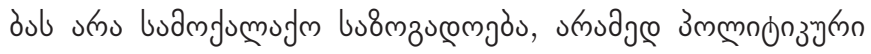

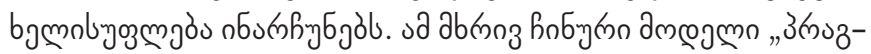

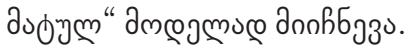

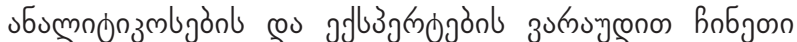

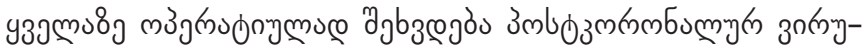

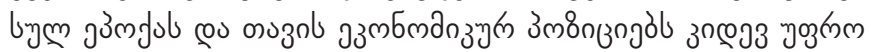

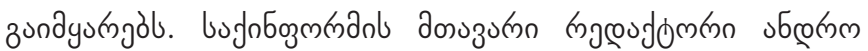

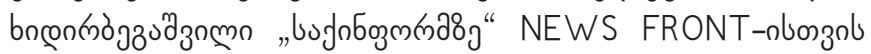

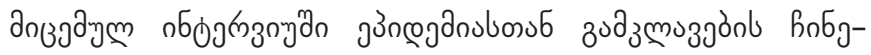

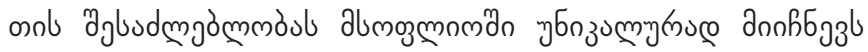

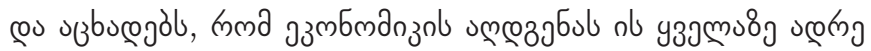
agdmgab.

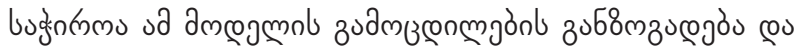

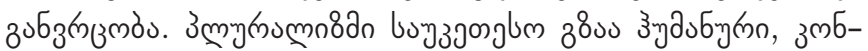

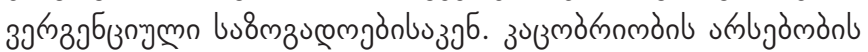

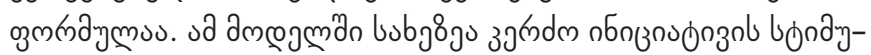

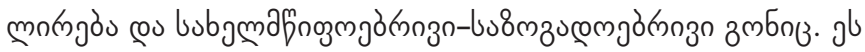

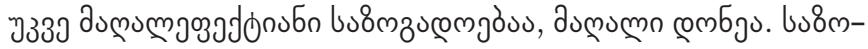

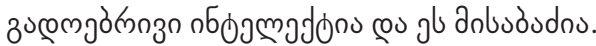

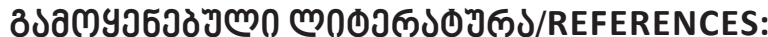

Bell, D. (1973). The coming of post-industrial society. A venture in social forecasting. New York

Kodua, E., et al. (Eds). (2004). Dictionary of Social and Political Terms. Lasha Beraia publication. Logo Press, Tbilisi, 351. (In Georgian).

Galbraith J. K. (1971). Exciting prospects. Za-Rubezho, 23, 4-10 / 6. (In Russian).

Gilman, N. (2020). China, Capitalism and the New Cold War. EPRC. (In Georgian). https://eprc.ge/index.php?m=11\&news_id=231 Hunt, E. K. \& Lautzenheiser, M. (2011). History of Economic Thought. A Critical Perspective. Armonk, New York; London England. Kharitonashvili, J. (1980). Theory of Convergence, GSE, 5. (In Georgian).

Kharitonashvili, J. (2019). History of Economic Thought. Tbilisi. (In Georgian).

Papava, V., \& Silagadze, A. (2019). How the Term the "Gross Domestic Product" should be Translated into Georgian. Globalization and Business, 7, 203-204. (In Georgian).

Saint-Simon, H. (1948). Selected works. 1-2, Moscow. (In Russian).

Skvortsov, V. N. (1974). Doctrine of Convergences and its Propaganda, Moscow. (In Russian).

Soros, J. (1997). The Threat of Capitalism. Literaturuli saqartvelo, 5.04-2.05 (In Georgian).

World history of economic thought (Editorial group) (1987) Moscow. (In Russian). 